\title{
Random walks on the BMW monoid: an algebraic approach
}

\author{
Sarah Wolff ${ }^{1}$ \\ Received: 30 March 2017 / Accepted: 9 November 2018 / Published online: 28 November 2018 \\ (c) Springer Science+Business Media, LLC, part of Springer Nature 2018
}

\begin{abstract}
We consider Metropolis-based systematic scan algorithms for generating BirmanMurakami-Wenzl (BMW) monoid basis elements of the BMW algebra. As the BMW monoid consists of tangle diagrams, these scanning strategies can be rephrased as random walks on links and tangles. We also consider the Brauer algebra and use Metropolis-based scans to generate Brauer diagrams, giving rise to random walks on perfect matchings. Taking an algebraic perspective, we translate these walks into left multiplication operators in the BMW algebra and so give an algebraic interpretation of the Metropolis algorithm in this setting.
\end{abstract}

Keywords Metropolis algorithm - Systematic scans · Random walks · Representation theory $\cdot$ Semisimple algebras

\section{Introduction}

Studying the convergence of random walks on finite groups, and in particular, the problem of generating group elements according to a fixed probability distribution has a long history $[6,9,11,22]$. Of particular interest for the purposes of this paper is the important work of Diaconis and Ram [10], who compare systematic scanning techniques with random scanning techniques in the context of generating elements of a finite Coxeter group $W$ using the Metropolis algorithm.

First introduced by Metropolis, Rosenbluth, Rosenbluth, Teller, and Teller [19], the Metropolis algorithm gives a method for sampling from a probability distribution $\pi$ by modifying an existing Markov chain to produce a new chain with stationary distribution $\pi$. This proves particularly useful for simulating configurations of particles with an associated energy (e.g., the influence that neighboring particles exert on each

Partially supported by the NSF GRFP under Grant No. DGE-1313911.

Sarah Wolff

wolffs@denison.edu

1 Denison University, Granville, USA 
other). Later applications of the Metropolis algorithm include the simulation of Ising models, initially developed to model a ferromagnet, but (surprisingly) also of use in image analysis and Gibbs sampling [5,12]. See [16] for additional applications. The Metropolis algorithm has the advantage of being straightforward to construct and implement; however, in analyzing the rate of convergence to $\pi$ rigorous bounds are often dependent on the specific situation. Further, these methods are most often examples of random scan Markov chains in that the process involved is that of selecting a site or set of sites to update at random. A more intuitively appealing and often more frequently used method in experimental work is that of a systematic scan Markov chain: a method to cycle through and update the sites in a deterministic order. While such scanning strategies may seem intuitive for use in sampling from $\pi$, they have proven difficult to analyze in many situations.

In [10] Diaconis and Ram use the Metropolis algorithm construction to produce Markov chains $K_{1}, K_{2}, \ldots, K_{n-1}$ corresponding to multiplication by the generators $r_{1}, \cdots, r_{n-1}$ of a Coxeter group $W$. For example, for each generator $r_{i}=(i i+1)$ of the symmetric group $S_{n}$, let

$$
Q_{i}(x, y)=\left\{\begin{array}{l}
1, \text { if } y=r_{i} x \\
0, \text { otherwise }
\end{array}\right.
$$

Then, for $l_{S}$ the length function on words in $S_{n}$, let $\pi$ be the probability distribution

$$
\pi(x)=\frac{\theta^{-l_{S}(x)}}{\sum_{w \in S_{n}} \theta^{-l_{S}(w)}} .
$$

The Metropolis algorithm construction, given explicitly in Sect. 2.2, modifies the chains $Q_{i}$ to produce Markov chains $K_{1}, K_{2}, \ldots, K_{n-1}$ that correspond to multiplication by the generators $r_{1}, \cdots, r_{n-1}$. For $K_{i}$ reversible, each with stationary distribution $\pi$, the following systematic scans produce reversible Markov chains with stationary distribution $\pi$ :

$$
\begin{array}{ll}
\frac{1}{n-1} \sum_{i=1}^{n-1} K_{i} & \text { (random scan), } \\
K_{1} K_{2} \cdots K_{n-1} K_{n-1} \cdots K_{2} K_{1} & \text { (short systematic scan), } \\
\left(K_{1} \cdots K_{n-1} K_{n-1} \cdots K_{1}\right) \cdots\left(K_{1} K_{2} K_{2} K_{1}\right)\left(K_{1} K_{1}\right) & \text { (long systematic scan). }
\end{array}
$$

In the context of generation of dihedral group and hypercube elements, Diaconis and Ram [10] show that convergence of the short systematic scan for the distribution $\pi$ above, with $l_{S}$ replaced by the length function on the group, occurs in the same number of steps as that of a random scan, i.e., choosing a random sequence of indices $\left\{i_{\ell}\right\}_{\ell=1}^{\infty}$. However, results for different scanning techniques or probability distributions remain open.

The key insight that allows for analysis of the Metropolis scans is the translation of the Markov chains $M_{i}$ into left multiplication operators in the corresponding 
Iwahori-Hecke Algebra. As generators of the Hecke algebra can be represented by braid diagrams, a natural extension is to consider this problem in the setting of the BMW algebra, whose generators can be represented by tangle diagrams (see Sect. 4.3). In this paper, we show that the methodology of [10] generalizes to the setting of random walks on the Brauer and BMW monoids, showing that the Metropolis walks on tangles and matchings are algebrized by the BMW algebra.

The Metropolis algorithm in this context gives rise to systematic scanning strategies for generating basis elements via multiplication by generators. The corresponding random walk on the BMW and Brauer monoids is defined in Sect. 3.2. We show that the random walk is given by left multiplication in the BMW algebra, allowing for the translation to the algebraic setting that is key to the methodology of [10]. For $B_{n}$ the set of Brauer diagrams on $n$ dots, $\left\{T_{r_{i}}, T_{e_{i}} \mid 1 \leq i \leq n-1\right\}$ generators of the BMW algebra, $\mathscr{T}_{n}$ a basis for the BMW algebra (determined in Sect. 4.3), and $\tilde{K}_{i}$ the matrix of the random walk we show

Theorem 1 Let $\mathscr{B} \mathscr{M} \mathscr{W}_{n}(m, l)$ be the BMW algebra with parameters $m=\theta^{-1}-1$ and $l=1$. Then,

$$
\sum_{y \in B_{n}} \tilde{K}_{i}\left(T_{d}, T_{y}\right) T_{y}= \begin{cases}T_{r_{i}} T_{d}, & \text { if } T_{r_{i}} T_{d} \in \mathscr{T}_{n} \\ \left(\theta T_{r_{i}}+(1-\theta) T_{e_{i}}\right) T_{d}, & \text { if } T_{r_{i}} T_{d} \notin \mathscr{T}_{n}\end{cases}
$$

After the algebraic translation of the problem, the main tools used in the analysis of [10] are a translation of the total variation norm into a trace norm on the Iwahori-Hecke algebra $H$. Rewriting this trace norm using characters of $H$ allows for bounds to come from the dimensions and traces of representations of $H$, rather than computation of the eigenvalues. Theorem 1 provides the necessary algebrization of the problem; in future work, we plan to similarly use a trace norm on the BMW algebra to bound the total variation norm by dimensions and traces of representations of the BMW algebra.

We begin in Sects. 2 and 3 with the preliminaries needed from probability theory and representation theory. We also give a presentation of the Brauer algebra. In Sect. 4, we describe the random walk arising from the Metropolis algorithm, give a presentation of the BMW algebra, and prove Theorem 1. We then conclude in Sect. 5 with further directions and questions.

\section{Preliminaries: Markov chains and the Metropolis algorithm}

We introduce the necessary probabilistic background, following the notation and outline of [10]. Further background can be found in $[1,4,15]$.

\subsection{Markov chains}

A Markov chain on a finite set $X$ is a matrix $K=(K(x, y))_{x, y \in X}$ such that $K(x, y) \in$ $[0,1]$ and 


$$
\text { if } x \in X \text { then } \sum_{y \in X} K(x, y)=1 \text {. }
$$

As a process on the finite set $X$, the value $K(x, y)$ gives the probability of moving from $x$ to $y$ in one step and $K^{m}(x, y)$ gives the probability of moving from $x$ to $y$ in $m$ steps. that

A stationary distribution for $K$ is a function $\pi: X \rightarrow[0,1], \sum_{x \in X} \pi(x)=1$ such

$$
\text { if } x \in X \text { then } \pi(x)=\sum_{y \in X} \pi(y) K(y, x) .
$$

A symmetric Markov chain is a Markov chain $K$ such that

$$
\text { if } x, y \in X \text { then } K(x, y)=K(y, x) \text {, }
$$

and a reversible Markov chain is a Markov chain $K$ such that

$$
\text { if } x, y \in X \text { then } \pi(x) K(x, y)=\pi(y) K(y, x) \text {. }
$$

An irreducible Markov chain is a Markov chain $K$ such that if $x, y \in X$, then there exists an integer $m$ such that $K^{m}(x, y)>0$. The Markov chain $K$ is aperiodic if

$$
\operatorname{gcd}\{m(x) \mid x \in X\}=1, \text { where } m(x)=\min \left\{m \in \mathbb{Z}_{>0} \mid K^{m}(x, x)>0\right\}
$$

for $x \in X$.

By the Perron-Frobenius theorem, irreducible aperiodic chains have a unique stationary distribution.

Let $K_{x}^{m}$ denotes the probability distribution $K^{m}(x, \cdot)$. The total variation distance from $K_{x}^{m}$ to $\pi$ is

$$
\left|K_{x}^{m}-\pi\right|_{T V}:=\max _{A \subseteq X}\left|\sum_{y \in A} K^{m}(x, y)-\pi(y)\right| .
$$

For $L^{2}(\pi)$ the space of functions $f: X \rightarrow \mathbb{R}$, equipped with the inner product

$$
\langle f, g\rangle_{2}=\sum f(x) g(x) \pi(x)
$$

the total variation distance is bounded by the $L^{2}(\pi)$ norm:

Lemma 1 (see, e.g., [10](Lemma 2.3)) For $f \in L^{2}(\pi)$,

$$
|f|_{T V}^{2} \leq \frac{1}{4}\|f / \pi\|_{2}^{2}
$$




\subsection{The Metropolis algorithm}

Let $P: X \times X \rightarrow[0,1]$ be a symmetric Markov chain, and let $\pi: X \rightarrow[0,1]$ be a probability distribution. Define a new Markov chain $K: X \times X \rightarrow[0,1]$ by

$$
K(x, y)= \begin{cases}P(x, y), & \text { if } x \neq y \text { and } \pi(y) \geq \pi(x), \\ P(x, y) \frac{\pi(y)}{\pi(x)}, & \text { if } x \neq y \text { and } \pi(y)<\pi(x), \\ P(x, x)+\sum_{\pi(z)<\pi(x)} P(x, z)\left(1-\frac{\pi(z)}{\pi(x)}\right), & \text { if } x=y .\end{cases}
$$

Metropolis, Rosenbluth, Rosenbluth, Teller, and Teller [19] shows that $K$ is a reversible Markov chain with stationary distribution $\pi$. Hammersley and Handscomb give a nice description of the Metropolis algorithm in [14].

While $K(x, y)$ has stationary distribution $\pi$, neither irreducibility nor aperiodicity is guaranteed. In particular, the Markov chains we consider in Sect. 3.2 are aperiodic, but not irreducible. To understand these chains, we consider their closed communication classes.

Definition 1 Let $K$ be a Markov chain with state space $X$. For $x, y \in X, y$ is accessible from $x$, denoted $x \rightarrow y$, if $K(x, y)^{n}>0$ for some $n \in \mathbb{Z}_{>0}$. $x$ communicates with $y$, denoted $x \leftrightarrow y$, if $x \rightarrow y$ and $y \rightarrow x$. The equivalence classes under the relation $\leftrightarrow$ are the communication classes of $K$. A communication class $C$ is closed if whenever $x \in C$ and $x \rightarrow y$ for some $y \in X$, then $y \in C$.

\section{Preliminaries: the Brauer algebra}

\subsection{Fourier inversion and Plancherel}

Let $A$ be a semisimple algebra, $\left\{a_{i}\right\}_{i \in I}$ a basis for $A$ and $f=\sum_{i \in I} f\left(a_{i}\right) a_{i} \in A$.

Definition 2 Let $\rho$ be a matrix representation of $A$. The Fourier transform of $f$ at $\rho$, denoted $\hat{f}(\rho)$, is the matrix sum

$$
\hat{f}(\rho)=\sum_{i \in I} f\left(a_{i}\right) \rho\left(a_{i}\right)
$$

For $G$ a finite group and $\mathbb{C}[G]$ the associated group algebra with basis the elements of $G$, we recover the Fourier transform of $G$ as studied in $[7,8,17]$. For more background on Fourier transforms in the semisimple algebra setting see $[10,18]$.

Random walks on groups are frequently studied using Fourier analysis. For example, for a finite group $G$ and $Q: G \rightarrow \mathbb{C}, \sum_{g \in G} Q(g)=1$ a probability distribution on $G$, let $\hat{Q}$ denotes the Fourier transform of $Q$. Let $U$ denotes the uniform distribution on $G$, i.e., $U(g)=\frac{1}{|G|}$ for each $g \in G$. The following theorem, specialized to the 


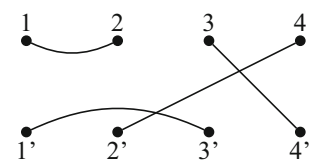

Fig. 1 A Brauer diagram $d$

finite group setting, can be found in [9]. The theorem in the semisimple algebra setting appears in [10].

Theorem 2 (Diaconis, [9]) For $G$ a finite group and $Q$ a probability distribution on $G$,

$$
|Q-U|_{T V}^{2} \leq \frac{1}{4} \sum_{\rho} d_{\rho} \operatorname{Tr}\left(\hat{Q}(\rho) \hat{Q}(\rho)^{*}\right)
$$

where * denotes conjugate transpose, and the sum is over all nontrivial irreducible representations $\rho$ of $G$.

A trace on a semisimple algebra $A$ is a $\mathbb{C}$-linear function $\tau: A \rightarrow \mathbb{C}$ such that if $a, b \in A$, then $\tau(a b)=\tau(b a)$. A trace function $\tau$ on $A$ gives rise to a symmetric bilinear form $\langle\cdot, \cdot\rangle_{\tau}: A \times A \rightarrow \mathbb{C}$ by letting

$$
\langle a, b\rangle_{\tau}=\tau(a b)
$$

for $a, b \in A$.

Further recall that for any trace function $\tau$ and set $R$ of inequivalent irreducible representations of $A$, there exist constants $t_{\rho} \in \mathbb{C}$ such that

$$
\tau=\sum_{\rho \in R} t_{\rho} \chi_{\rho},
$$

where for $a \in A, \chi_{\rho}(a)=\operatorname{Tr}(\rho(a))$.

\subsection{The Brauer algebra}

Let $n$ be a positive integer and $q$ an indeterminate. A Brauer diagram on $n$ dots is a graph on $2 n$ vertices $\left\{1, \ldots, n, 1^{\prime} \ldots, n^{\prime}\right\}$ with edges partitioning the vertices into pairs (Fig. 1).

Edges that pair two dots on the same row are arcs. The set $B_{n}$ of Brauer diagrams on $n$ dots has cardinality $(2 n-1) ! !=(2 n-1)(2 n-3) \cdots 5 \cdot 3 \cdot 1$.

Let $x$ and $y$ be two Brauer diagrams on $n$ dots. Place $x$ above $y$ and identify the vertices in the bottom row of $x$ with those in the top row of $y$. Let $c$ be the number of closed loops obtained in this concatenation. Then, the product $x y$ is given by $q^{c}$ times the resulting diagram without loops (Fig. 2).

The Brauer algebra $\mathscr{B} r_{n}(q)$ is the $\mathbb{C}[q]$-span of the Brauer diagrams under the $\mathbb{C}$-linear extension of diagram multiplication. The Brauer algebra is generated by 


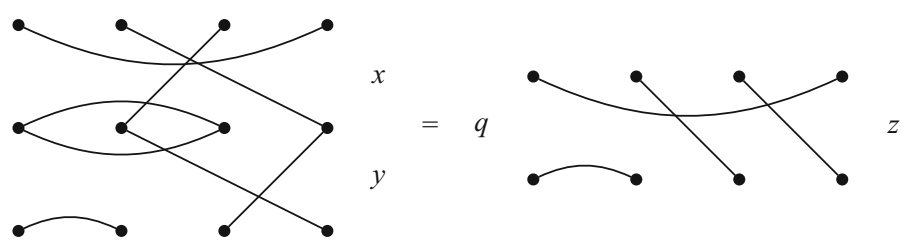

Fig. $2 x y=q^{1} z$

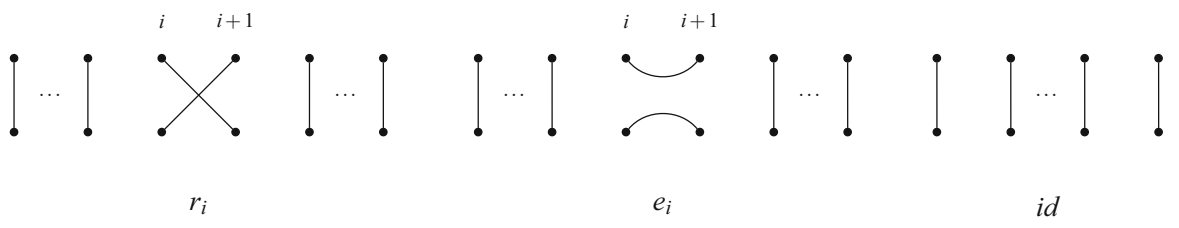

Fig. $3 r_{i}, e_{i}, i d \in B r_{n}$

$\left\{r_{i}, e_{i} \mid 1 \leq i \leq n-1\right\}$, for $r_{i}$ and $e_{i}$ as in Fig. 3. The identity element of $\mathscr{B} r_{n}(q)$ is given in Fig. 3.

Benkart, Shader, and Ram note in [2] that the following presentation of the Brauer algebra is "'oft-quoted,' (see, for example, [21]), but we are not aware of a proof in the literature. In the interests of space, we shall continue the tradition and not include a proof here either." We follow their example and note the following presentation of the Brauer algebra.

Theorem 3 The Brauer algebra $\mathscr{B} r_{n}$ has presentation given by generating set $S=$ $\left\{r_{i}, e_{i} \mid 1 \leq i \leq n-1\right\}$ and relations
(B1) $r_{i}^{2}=1$,
(B2) $r_{i} r_{j}=r_{j} r_{i}, \quad r_{i} e_{j}=e_{j} r_{i}, \quad e_{i} e_{j}=e_{j} e_{i}$,
(B3) $e_{i}^{2}=q e_{i}$, $|i-j|>1$
(B5) $r_{i} r_{i+1} r_{i}=r_{i+1} r_{i} r_{i+1}$,
(B4) $e_{i} r_{i}=r_{i} e_{i}=e_{i}$,
(B7) $r_{i} e_{i+1} e_{i}=r_{i+1} e_{i}$,
(B6) $e_{i} e_{i+1} e_{i}=e_{i}, \quad e_{i+1} e_{i} e_{i+1}=e_{i+1}$,
(B8) $e_{i+1} e_{i} r_{i+1}=e_{i+1} r_{i}$.

\section{Length in $B_{n}$ and the walk}

\subsection{Reduced expressions and length}

Definition $3 \mathrm{~A}$ word $W=s_{i_{1}} \ldots s_{i_{n}}$ with $s_{i_{j}} \in S$ has an occurrence of $e_{i+1} r_{i}$ if a subword of $W$ has form $w=e_{i+1} s_{j_{1}} \ldots s_{j_{k}} r_{i}$, where $j_{i}, \ldots, j_{k} \neq i, i+1$.

By Brauer relation (B2), the subword $w$ can be rewritten $w=s_{i_{1}} \ldots s_{i_{l}} e_{i+1} r_{i} s_{i_{l+1}} \ldots s_{i_{k}}$ for some $1 \leq l \leq k$. We remove the occurrence of $e_{i+1} r_{i}$ using Brauer relation (B8); replace $w$ with $w^{\prime}=s_{i_{1}} \ldots s_{i_{l}} e_{i+1} e_{i} r_{i+1} s_{i_{l+1}} \ldots s_{i_{k}}$.

Example 1 Let $W=e_{6} e_{3} r_{4} r_{5} e_{1} r_{2}$, which has an occurrence of $e_{3} r_{2}: w=e_{3} r_{4} r_{5} e_{1} r_{2}$. By Brauer relation $(B 2), w=e_{3} e_{1} r_{4} r_{5} r_{2}=e_{1} e_{3} r_{2} r_{4} r_{5}$. Replace $w$ with $w^{\prime}=$ $e_{1} e_{3} e_{2} r_{3} r_{4} r_{5}$. Note that $W=e_{6} e_{1} e_{3} e_{2} r_{3} r_{4} r_{5}$ has no occurrence of $e_{3} r_{2}$. 


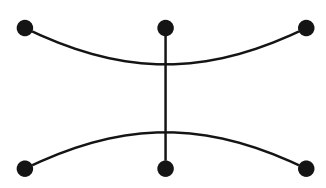

Fig. $4 r_{2} e_{1} r_{2}$

The $e_{i+1}$ term in $w^{\prime}$ cannot form a new occurrence of $e_{i+1} r_{i}$ in $W$ as it has $e_{i}$ directly to its right. The $e_{i}$ term, however, could form a new occurrence of $e_{i} r_{i-1}$ in $W$.

Example 2 Let $W=e_{3} r_{4} e_{5} r_{2} r_{1}$, which has an occurrence of $e_{3} r_{2}$ through subword $w=e_{3} r_{4} e_{5} r_{2}=e_{3} r_{2} r_{4} e_{5}$. We remove this occurrence by replacing $w$ with $w^{\prime}=$ $e_{3} e_{2} r_{3} r_{4} e_{5}$. Then $W=e_{3} e_{2} r_{3} r_{4} e_{5} r_{1}$, which has an occurence of $e_{2} r_{1}$.

As replacing an occurrence of $e_{i+1} r_{i}$ can at worst create a new occurrence of $e_{i} r_{i-1}$, the process of removing all possible occurrences of $e_{i+1} r_{i}, 1 \leq i \leq n-2$ from $W$ terminates after finitely many removals.

Example 3 Let $W=e_{3} r_{4} e_{5} r_{2} r_{1}$ as in Example 2. After removing the occurrence of $e_{3} r_{2}, W=e_{3} e_{2} r_{3} r_{4} e_{5} r_{1}$, with subword $w=e_{2} r_{3} r_{4} e_{5} r_{1}=e_{2} r_{1} r_{3} r_{4} e_{5}$. Replacing $w$ with $w^{\prime}=e_{2} e_{1} r_{2} r_{3} r_{4} e_{5}$ yields $W=e_{3} e_{2} e_{1} r_{2} r_{3} r_{4} e_{5}$ which has no occurrence of $e_{i+1} r_{i}$ for any $i$.

Definition 4 Let $d \in B_{n}$. A reduced expression for $d$ is a word $d=s_{i_{1}} \cdots s_{i_{n}}$ with no occurrences of $e_{i+1} r_{i}$ for all $1 \leq i \leq n-2$.

Example 4 Let $d=r_{2} e_{1} r_{2} \in B_{3}$ be the diagram of Fig. 4. This expression for $d$ has no occurrence of $e_{2} r_{1}$, so it is a reduced expression for $d$. Note that another expression for $d$ is given by $d=r_{1} e_{2} r_{1}$; however, this is not a reduced expression for $d$ as it has an occurrence of $e_{2} r_{1}$. Indeed, removing the occurrence of $e_{2} r_{1}$ yields $d=r_{1} e_{2} e_{1} r_{2}=r_{2} e_{1} r_{2}$, with the last equality due to Brauer relation (B7).

Define the length of a word $s_{i_{1}} \cdots s_{i_{n}}$ to be

$$
l\left(s_{i_{1}} \cdots s_{i_{n}}\right)=\#\left\{r_{i} \text { terms }\right\}+2 \#\left\{e_{i} \text { terms }\right\} .
$$

Define the length function on the set of Brauer diagrams $L: B_{n} \rightarrow \mathbb{Z}_{>0}$ by

$$
L(d)=\min \{l(W) \mid W \text { a reduced expression for } d\}
$$

Example 5 Let $d=r_{2} e_{1} r_{2}$ as in Fig. 4. Then $L(d)=4$. From this example, it may seem that the length function gives the number of crossings of $d$ plus the number of $\operatorname{arcs}$ of $d$; however, this is not the case. For example, the element $d^{\prime}=e_{1} e_{2}$ has two arcs and zero crossings, but $l\left(d^{\prime}\right)=4$ (Fig. 5). 


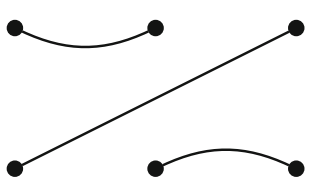

Fig. $5 d^{\prime}=e_{1} e_{2}$
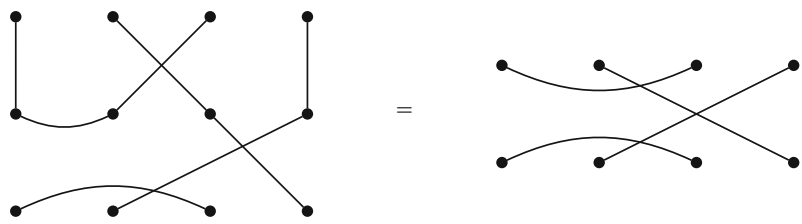

Fig. $6 r_{2} d$

\subsection{The walk}

The symmetric group $S_{n}$ acts on the set $B_{n}$ of Brauer diagrams by permuting the vertices $1, \ldots, n$. Let $r_{i}$ be the transposition in $S_{n}$ that switches vertex $i$ with vertex $i+1$. Then $r_{i}$ is as in Fig. 3, with the action $r_{i} d$ given by the multiplication $r_{i} d$ in the Brauer algebra. (see Fig. 6).

Let $\theta \in[0,1]$ and define a distribution $\pi: B_{n} \rightarrow[0,1]$ by

$$
\pi(d)=\frac{1}{Z} \theta^{-L(d)}, \quad \text { where } Z=\sum_{z \in B_{n}} \theta^{-L(z)} .
$$

Define $P_{i}: B_{n} \times B_{n} \rightarrow[0,1]$ by

$$
P_{i}(d, y)= \begin{cases}1, & \text { if } y=r_{i} d \\ 0, & \text { if } y \neq r_{i} d\end{cases}
$$

As $r_{i}^{-1}=r_{i}, P(d, y)=P(y, d)$ for all $d, y \in B_{n}$. The chain $P_{i}$ corresponds to the simple random walk on the graph with vertices labeled by diagrams $d \in B_{n}$ and edge set $\left\{\{d, y\} \mid y=r_{i} d\right\}$.

Applying the Metropolis algorithm (3) to $P_{i}$ and $\pi$ yields $K_{i}: B_{n} \times B_{n} \rightarrow[0,1]$ where

$$
K_{i}(d, y)= \begin{cases}1, & \text { if } y=r_{i} d \text { and } L(d) \leq L(y), \\ \theta, & \text { if } y=r_{i} d \text { and } L(d)>L(y), \\ 1-\theta, & \text { if } y=d \text { and } L(d)>L\left(r_{i} d\right), \\ 0, & \text { otherwise }\end{cases}
$$

In Appendix A, we give an example of the chain $K_{1}: B_{3} \times B_{3} \rightarrow[0,1]$. Interpreted as a random walk on $B_{n}$, the chain $K_{i}$ describes the process:

From $d \in B_{n}$ act on the left by $r_{i}$. If the length increases,

move to $r_{i} d$. If the length decreases, flip a $\theta$-coin and if heads move to $r_{i} d$. If tails, remain at $d$. 


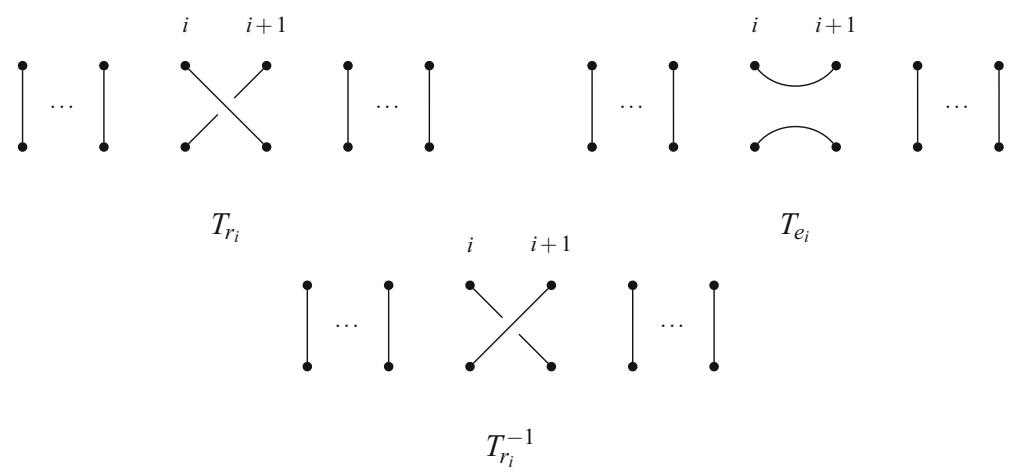

Fig. $7 T_{r_{i}}, T_{e_{i}}, T_{r_{i}}^{-1}$
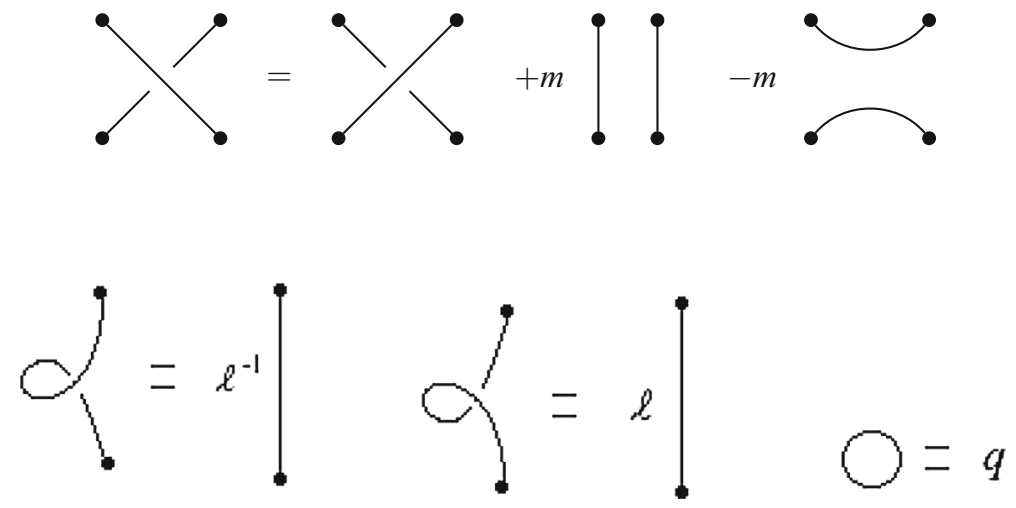

Fig. 8 Untangling relations

Note 1 Note that $S_{n} \subset B_{n}$ as $S_{n}$ is the set of Brauer diagrams without arcs. For $d \in S_{n}$, $L(d)=\ell(d)$ for $\ell: S_{n} \rightarrow \mathbb{Z}$ the length function on $S_{n}$ that gives the minimum number of transpositions $r_{i}$ needed to express $d$. The submatrix of $K_{i}$ corresponding to $\left\{d \mid d \in S_{n}\right\}$ is exactly the chain $M_{i}$ of [10].

\subsection{The BMW algebra}

For $m, \ell, q$ parameters satisfying $q=\left(\ell-\ell^{-1}\right)\left(m-m^{-1}\right)^{-1}+1$, the BMW algebra $\mathscr{B} \mathscr{M} \mathscr{W}_{n}(m, l)$ is given by generating set $\left\{T_{e_{i}}, T_{r_{i}}, T_{r_{i}}^{-1} \mid 1 \leq i \leq n-1\right\}$ and relations
(A1) $T_{r_{i}}=T_{r_{i}}^{-1}+m T_{i d}-m T_{e_{i}}$,
(A2) $T_{r_{i}}^{ \pm 1} T_{r_{j}}^{ \pm 1}=T_{r_{j}}^{ \pm 1} T_{r_{i}}^{ \pm 1}, \quad T_{r_{i}}^{ \pm 1} T_{e_{j}}=T_{e_{j}} T_{r_{i}}^{ \pm 1}$,
(A3) $T_{e_{i}}^{2}=q T_{e_{i}}$, $T_{e_{i}} T_{e_{j}}=T_{e_{j}} T_{e_{i}}, \quad|i-j|>1$,
(A5) $T_{r_{i}} T_{r_{i+1}} T_{r_{i}}=T_{r_{i+1}} T_{r_{i}} T_{r_{i+1}}$,
(A4) $T_{e_{i}} T_{r_{i}}=T_{r_{i}} T_{e_{i}}=\ell^{-1} T_{e_{i}}$
(A7) $T_{r_{i}} T_{r_{i \pm 1}} T_{e_{i}}=T_{e_{i \pm 1}} T_{e_{i}}=T_{e_{i \pm 1}} T_{r_{i}} T_{r_{i \pm 1}}$,
(A6) $T_{e_{i}} T_{e_{i \pm 1}} T_{e_{i}}=T_{e_{i}}$,
(A8) $T_{e_{i}} T_{r_{i \pm 1}} T_{e_{i}}=\ell T_{e_{i}}$,

(see, for example [13]). The standard pictorial representation of the generators is given in Fig. 7 and, pictorially, the relations are (Fig. 8). 
Fig. 9 Lifts of $r_{2} e_{1} r_{2}$
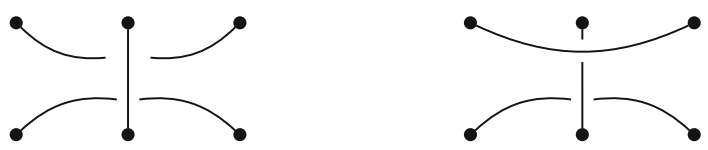

Let $d \in B_{n}$. An element of $\mathscr{B} \mathscr{M} \mathscr{W}_{n}(m, l)$ is a lift of $d$ if it is achieved by drawing $d$ so that for each crossing of $d$, one edge is drawn to pass above the other. See Fig. 9 for two of the four possible lifts of $d=r_{2} e_{1} r_{2}$.

Given $d \in B_{n}$, choose a sequence of nodes of $d$ consisting of one end point of each edge. An element of $\mathscr{B} \mathscr{M} \mathscr{W}_{n}(m, l)$ is totally descending (with this choice of nodes) if it is the result of drawing each edge of $d$ in order, so that when meeting an edge that has already been drawn, the new edge crosses under the existing one.

Theorem 4 (Morton, Wasserman [20]) $\mathscr{B} \mathscr{M} \mathscr{W}_{n}(m, l)$ is spanned by its totally descending elements.

Note that this holds for any fixed choice of sequence of nodes of $d$, and thus, $\mathscr{B} \mathscr{M}^{\mathscr{W}}{ }_{n}(m, l)$ is spanned by any fixed set of lifts of $B_{n}$ to $\mathscr{B} \mathscr{M} \mathscr{W}_{n}(m, l)$.

For a Brauer diagram $d \in B_{n}$, let $d=s_{i_{1}} \cdots s_{i_{n}}$ be a reduced expression for $d$ of minimal length and let

$$
T_{d}=T_{s_{i_{1}}} \cdots T_{s_{i_{n}}}
$$

This is well defined as the only length-preserving relations in the Brauer algebra are (B2) and (B5) and these relations hold in the BMW algebra as (A2) and (A5). Let $\mathscr{T}_{n}=\left\{T_{d} \mid d \in B_{n}\right\}$. By Theorem $4, \mathscr{T}_{n}$ spans $\mathscr{B} \mathscr{M} \mathscr{W}_{n}(m, l)$.

Lemma 2 Let $B M W_{n}(m, l)$ be the BMW algebra with parameter $l=1$. Let $T_{d} \in \mathscr{T}_{n}$. Then

$$
L\left(r_{i} d\right)<L(d) \Longleftrightarrow T_{r_{i}} T_{d} \notin \mathscr{T}_{n}
$$

and in this case $T_{r_{i}}^{-1} T_{d}=T_{r_{i} d}$.

Proof Let $d=s_{i_{1}} \cdots s_{i_{k}} \in B_{n}$.

Case 1: $L\left(r_{i} d\right)>L(d)$

As multiplication by $r_{i}$ cannot introduce an occurrence of $e_{j} r_{j-1}$, if $L\left(r_{i} d\right)>$ $L(d)$, then $L\left(r_{i} d\right)=L(d)+1$ and $r_{i} s_{i_{1}} \cdots s_{i_{k}}$ is a reduced expression for $r_{i} d$. Thus, $T_{r_{i}} T_{d}=T_{r_{i}} T_{s_{i_{1}}} \cdots T_{s_{i_{k}}}=T_{r_{i} d}$.

Case 2: $L\left(r_{i} d\right)=L(d)$

As Brauer relation $(B 4)$ is the only length-preserving multiplication by $r_{i}$, if $L\left(r_{i} d\right)=L(d)$, then $r_{i} d=s_{i_{1}} \cdots r_{i} e_{i} s_{i_{j+1}} \cdots s_{i_{k}}$ for some $1 \leq j \leq k$, with $\left|i_{l}-i\right|>1$ for $l<j$ (using Brauer relation (B2)). By Brauer relation $(B 4), r_{i} d=d$ and so a reduced expression for $r_{i} d$ is $s_{i_{1}} \cdots s_{i_{k}}$. By BMW relations (A2) and (A4),

$$
T_{r_{i}} T_{d}=T_{r_{i}} T_{s_{i_{1}}} \cdots T_{s_{i_{k}}}=T_{s_{i_{1}}} \cdots T_{r_{i}} T_{e_{i}} T_{s_{i_{j+1}}} \cdots T_{s_{i_{k}}}=l^{-1} T_{d}
$$

As $l^{-1}=1$ and $r_{i} d=d, T_{r_{i}} T_{d}=T_{r_{i} d}$. 
Case 3: $L\left(r_{i} d\right)<L(d)$

As Brauer relation $(B 1)$ and Brauer relation $(B 7)$ give the only possibilities for multiplication by $r_{i}$ to reduce length, if $L\left(r_{i} d\right)<L(d)$ then $r_{i} d=s_{i_{1}} \cdots r_{i} r_{i} s_{i_{j+1}} \cdots s_{i_{k}}$ for some $1 \leq j \leq k$ with $\left|i_{l}-i\right|>1$ for $l<j$ or $r_{i} d=s_{i_{1}} \cdots r_{i} e_{i+1} e_{i} s_{i_{j+2}} \cdots s_{i_{k}}$ for some $1 \leq j \leq k-1$ with $\left|i_{l}-i\right|>1$ for $l<j$.

Case 3a: $r_{i} d=s_{i_{1}} \cdots r_{i} r_{i} s_{i_{j+1}} \cdots s_{i_{k}}$

Then by Brauer relation $(B 1), r_{i} d=s_{i_{1}} \cdots s_{i_{j-1}} s_{i_{j+1}} \cdots s_{i_{k}}$. In particular, $r_{i} s_{i_{1}} \cdots s_{i_{k}}$ is not a minimal length expression for $r_{i} d$. As Brauer relation $(B 1)$ does not hold in the BMW algebra,

$$
T_{r_{i}} T_{d}=T_{s_{i_{1}}} \cdots T_{r_{i}} T_{r_{i}} T_{s_{i_{j+1}}} \cdots T_{s_{i_{k}}} \neq T_{s_{i_{1}}} \cdots T_{s_{i_{j-1}}} T_{s_{i_{j+1}}} \cdots T_{s_{i_{k}}},
$$

and so $T_{r_{i}} T_{d} \neq T_{r_{i} d}$. Note, however, that

$$
T_{r_{i}}^{-1} T_{d}=T_{s_{i_{1}}} \cdots T_{r_{i}}^{-1} T_{r_{i}} T_{s_{i_{j+1}}} \cdots T_{s_{i_{k}}}=T_{r_{i} d}
$$

Case 3b: $r_{i} d=s_{i_{1}} \cdots r_{i} e_{i+1} e_{i} s_{i_{j+2}} \cdots s_{i_{k}}$

As in the previous case, $r_{i} s_{i_{1}} \cdots s_{i_{k}}$ is not a minimal length expression for $r_{i} d$ as $r_{i} d=s_{i_{1}} \cdots r_{i} e_{i+1} e_{i} s_{i_{j+2}} \cdots s_{i_{k}}=s_{i_{1}} \cdots r_{i+1} e_{i} s_{i_{j+2}} \cdots s_{i_{k}}$ (using Brauer relation (B7)). Brauer relation (B7) does not hold in the BMW algebra, so $T_{r_{i}} T_{d} \neq T_{r_{i} d}$. Note, however, that by BMW relation (A7) $T_{r_{i}}^{-1} T_{e_{i+1}} T_{e_{i}}=T_{r_{i}}^{-1} T_{r_{i}} T_{r_{i+1}} T_{e_{i}}=$ $T_{r_{i+1}} T_{e_{i}}$, and so $T_{r_{i}}^{-1} T_{d}=T_{r_{i} d}$.

For $K_{i}$ the Markov chain defined by Equation (5), define $\tilde{K}_{i}: \mathscr{T}_{n} \times \mathscr{T}_{n} \rightarrow[0,1]$ by

$$
\tilde{K}_{i}\left(T_{d}, T_{y}\right)=K_{i}(d, y)
$$

We now prove Theorem 1, giving an interpretation of the Markov chains $K_{i}$ in terms of multiplication in the $B M W$ algebra.

Theorem 5 1.1 Fix $\theta \in[0,1]$ and $i \in\{1, \ldots, n-1\}$. Let $\mathscr{B} \mathscr{M}^{\mathscr{W}}{ }_{n}(m, l)$ be the BMW algebra with parameters

$$
m=\theta^{-1}-1 \text { and } l=1 .
$$

Then

$$
\sum_{y \in B_{n}} \tilde{K}_{i}\left(T_{d}, T_{y}\right) T_{y}= \begin{cases}T_{r_{i}} T_{d}, & \text { if } T_{r_{i}} T_{d} \in \mathscr{T}_{n} \\ \left(\theta T_{r_{i}}+(1-\theta) T_{e_{i}}\right) T_{d}, & \text { if } T_{r_{i}} T_{d} \notin \mathscr{T}_{n}\end{cases}
$$

Proof Suppose $T_{r_{i}} T_{d} \in \mathscr{T}_{n}$. Then by Lemma 2, $L\left(r_{i} d\right) \geq L(d)$, meaning that $\tilde{K}_{i}\left(T_{d}, T_{y}\right)=0$ for $y \neq r_{i} d$ and $\tilde{K}_{i}\left(T_{d}, T_{r_{i} d}\right)=1$. Since $T_{r_{i}} T_{d} \in \mathscr{T}_{n}, T_{r_{i}} T_{d}=$ $T_{d^{\prime}}=T_{s_{i_{1}}} \cdots T_{s_{i_{n}}}$ for some reduced expression $s_{i_{1}} \cdots s_{i_{n}}=r_{i} d$ of $d^{\prime}=r_{i} d \in B_{n}$ and so $T_{r_{i}} T_{d}=T_{r_{i} d}$ 
Now suppose $T_{r_{i}} T_{d} \notin \mathscr{T}_{n}$. Then by Lemma $2, L\left(r_{i} d\right)<L(d)$, meaning that

$$
\sum_{y \in B_{n}} \tilde{K}_{i}\left(T_{d}, T_{y}\right) T_{y}=\theta T_{r_{i} d}+(1-\theta) T_{d}
$$

Also by Lemma $2, T_{r_{i}}^{-1} T_{d}=T_{r_{i} d}$ and so by BMW relation $(A 1)$,

$$
\theta T_{r_{i}} T_{d}=\theta\left(T_{r_{i}}^{-1} T_{d}+m T_{d}-m T_{e_{i}} T_{d}\right)=\theta T_{r_{i} d}+(1-\theta) T_{d}-(1-\theta) T_{e_{i}} T_{d} .
$$

Adding $(1-\theta) T_{e_{i}} T_{d}$ gives the desired result.

\section{Further directions}

In this paper, we extend the methodology of [10] to consider random walks on the BMW monoid arising from the Metropolis algorithm. We provide an algebraic translation of the problem, showing that Metropolis walks on the Brauer and BMW monoids are algebrized by the BMW algebra. An important next step would be to perform the mixing time analysis and use it to compare different scanning techniques.

We provide algebraic methods and tools for studying Metropolis-based random walks on the BMW monoid. As the BMW algebra is but one of a wealth of examples of diagram algebras, it would be interesting to explore Metropolis-based walks on other diagrams, as well as different random walks encoding statistics on these diagrams. For example, random walks on subalgebras of $\mathbb{C}\left[S_{n}\right]$ have been used to study permutation statistics [3]. Such walks could be extended to study statistics on perfect matchings and partitions. As the Brauer algebra (respectively, the partition algebra) is generated by diagrams encoding perfect matchings (respectively, set partitions), algebraic translation of such walks would allow for the use of the representation theory of the Brauer and partition algebras to study these statistics.

Acknowledgements The author would like to especially thank Arun Ram for his interest in the work and many helpful conversations, as well as Daniel Rockmore for his guidance and support. The author would also like to thank two anonymous referees for the many helpful suggestions that greatly improved the paper.

\section{A Example of the walk in $\mathscr{B} \mathscr{M} \mathscr{W}_{3}$}

Example 6 In $\mathscr{B} \mathscr{M} \mathscr{W}_{3}$,

$$
\mathscr{B}_{3}=\mathbf{R} \cup \mathbf{E}_{1} \cup \mathbf{E}_{2} \cup \mathbf{E}_{3},
$$

for

$$
\begin{array}{ll}
\mathbf{R}=\left\{T_{i d}, T_{r_{1}}, T_{r_{2}}, T_{r_{1}} T_{r_{2}}, T_{r_{2}} T_{r_{1}}, T_{r_{1}} T_{r_{2}} T_{r_{1}}\right\}, & \mathbf{E}_{1}=\left\{T_{e_{1}}, T_{r_{2}} T_{e_{1}}, T_{e_{2}} T_{e_{1}}\right\}, \\
\mathbf{E}_{2}=\left\{T_{e_{2}}, T_{r_{1}} T_{e_{2}}, T_{e_{1}} T_{e_{2}}\right\}, & \mathbf{E}_{3}=\left\{T_{e_{1}} T_{r_{2}}, T_{r_{2}} T_{e_{1}} T_{r_{2}}, T_{e_{2}} T_{e_{1}} T_{r_{2}}\right\} .
\end{array}
$$


The Markov chain $K_{1}$ has form

$$
R \bigoplus E_{1} \bigoplus E_{2} \bigoplus E_{3}
$$

for

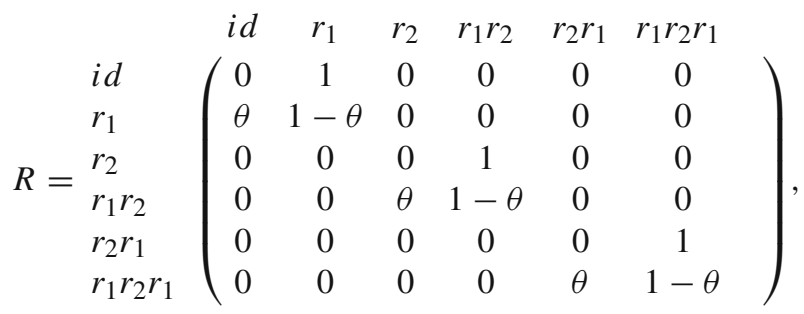

$$
\begin{aligned}
& E_{1}=\begin{array}{ccc}
e_{1} & r_{2} e_{1} & e_{2} e_{1} \\
e_{1} e_{1} \\
e_{2} e_{1}
\end{array}\left(\begin{array}{ccc}
1 & 0 & 0 \\
0 & 0 & 1 \\
0 & \theta & 1-\theta
\end{array}\right), E_{2}=\begin{array}{l}
e_{2} \\
r_{1} e_{2} \\
e_{1} e_{2}
\end{array}\left(\begin{array}{cccc}
e_{2} & r_{1} e_{2} & e_{1} e_{2} \\
\theta & 1-\theta & 0 \\
0 & 0 & 1
\end{array}\right), \\
& \begin{array}{lll}
e_{1} r_{2} & r_{2} e_{1} r_{2} & e_{2} e_{1} r_{2}
\end{array} \\
& E_{3}=\begin{array}{l}
e_{1} r_{2} \\
r_{2} e_{1} r_{2} \\
e_{2} e_{1} r_{2}
\end{array}\left(\begin{array}{ccc}
1 & 0 & 0 \\
0 & 0 & 1 \\
0 & \theta & 1-\theta
\end{array}\right) .
\end{aligned}
$$

\section{References}

1. Aldous, D., Fill, J.A.: Reversible markov chains and random walks on graphs. Unfinished monograph, recompiled 2014 (2002).http://www.stat.berkeley.edu/ aldous/RWG/book.html

2. Benkart, G., Ram, A., Shader, C.L.: Tensor product representations for orthosymplectic Lie superalgebras. J. Pure Appl. Algebra 130(1), 1-48 (1998)

3. Bergeron, F., Favreau, L.: Fourier transform over semi-simple algebras and harmonic analysis for probabilistic algorithms. Discrete Math., 139(1-3):19-32. Formal power series and algebraic combinatorics (Montreal, PQ, 1992) (1995)

4. Brémaud, P.: Markov chains, volume 31 of texts in applied mathematics. In: Marsden, J.E., Sirovich, L., Golubitsky, M., Jäger, W. (eds.) Gibbs fields, Monte Carlo simulation, and queues. Springer, New York (1999)

5. Cai, Y.: How rates of convergence for Gibbs fields depend on the interaction and the kind of scanning used. In: Hou, Z., Filar, J.A., Chen, A. (eds.) Markov Processes and Controlled Markov Chains (Changsha, 1999), pp 489-498. Kluwer Acad. Publ, Dordrecht (2002)

6. Ceccherini-Silberstein, T., Scarabotti, F., Tolli, F.: Harmonic Analysis on Finite Groups, Volume 108 of Cambridge Studies in Advanced Mathematics. Representation Theory, Gelfand Pairs and Markov Chains. Cambridge University Press, Cambridge (2008)

7. Clausen, M.: Fast generalized Fourier transforms. Theoret. Comput. Sci. 67(1), 55-63 (1989)

8. Clausen, M., Baum, U.: Fast Fourier Transforms. Bibliographisches Institut, Mannheim (1993)

9. Diaconis, P.: Group representations in probability and statistics. Institute of Mathematical Statistics Lecture Notes-Monograph Series, 11. Institute of Mathematical Statistics., Hayward, CA (1988)

10. Diaconis, P., Ram, A.: Analysis of systematic scan Metropolis algorithms using Iwahori-Hecke algebra techniques. Michigan Math. J. 48, 157-190 (2000) 
11. Diaconis, P., Saloff-Coste, L.: Random walks on finite groups: a survey of analytic techniques. In: Heyer, H. (ed.) Probability Measures on Groups and Related Structures. XI (Oberwolfach, 1994), pp. 44-75. World Sci. Publ, River Edge (1995)

12. Fishman, G.: Coordinate selection rules for Gibbs sampling. Ann. Appl. Probab. 6(2), 444-465 (1996)

13. Goodman, F., Hauschild, H.: Affine Birman-Wenzl-Murakami algebras and tangles in the solid torus. Fundam. Math. 190, 77-137 (2006)

14. Hammersley, J.M., Handscomb, D.C.: Monte Carlo Methods. Methuen \& Co., Ltd., Barnes \& Noble, Inc., London, New York (1965)

15. Karlin, S.: A First Course in Stochastic Processes. Academic Press, New York (1966)

16. Liu, J.: Monte Carlo Strategies in Scientific Computing. Springer Series in Statistics. Springer, New York (2008)

17. Maslen, D., Rockmore, D.: Separation of variables and the computation of Fourier transforms on finite groups. I. J. Amer. Math. Soc. 10(1), 169-214 (1997)

18. Maslen, D., Rockmore, D.N., Wolff, S.: The efficient computation of fourier transforms on semisimple algebras. J. Fourier Anal. Appl. 24, 1377-1400 (2017)

19. Metropolis, N., Rosenbluth, A., Rosenbluth, M., Teller, A., Teller, E.: Equation of state calculations by fast computing machines. J. Chem. Phys. 21(1087), 1087-1092 (1953)

20. Morton, H., Wasserman, A.: A basis for the Birman-Wenzl algebra. Revised 2000. Unpublished Manuscript (1989)

21. Nazarov, M.: Young's orthogonal form for Brauer's centralizer algebra. J. Algebra 182(3), 664-693 (1996)

22. Saloff-Coste, L.: Random walks on finite groups. In: Kesten, H. (ed.) Probability on Discrete Structures, Volume 110 of Encyclopaedia Math. Sci., pp. 263-346. Springer, Berlin (2004) 